\title{
Optimization Methods and Algorithms for Solving Of Hydro- Thermal Scheduling Problems.
}

\author{
G.N. Ajah and B.O. Anyaka \\ Department of Electrical Engineering, University of Nigeria, Nsukka, Nigeria.
}

\begin{abstract}
Different optimization methods have been applied to solve hydrothermal scheduling problems. This study reviews some of the common optimization methods and algorithms their strengths and weaknesses. The study found out that with time, old methods are improved upon and novel methods are developed to provide for more efficiency, faster convergence, robustness, and adaptability.
\end{abstract}

Keywords: Optimization, ALM, DE, FAPSO, EDDP, Hydrothermal

\section{Introduction}

Optimal scheduling of power plant generation is the determination of the generation for every generating unit such that the total system generation cost is minimum while satisfying the system constraints [1]. All hydro-systems are unique in their characteristics. Natural differences in water areas, difference between release elements, control constraints, non-uniform water flow, sudden alterations in the volume of water flow due to seasonal or natural constraints, occurrence of flood, drought and other natural phenomenon are among factors that affect hydro scheduling. The objective of the hydrothermal scheduling problem is to determine the water releases from each reservoir of the hydro system at each stage such that the operation cost is minimized along the planning period [1].

The importance of hydrothermal generation scheduling is well recognized. An efficient generation schedule not only reduces the production costs but also increases the system reliability and maximizes the energy capability of the reservoirs [2].Therefore, many methods have been developed to solve this problem over the past decades. The major methods include variational calculus [3], maximum principle [4], functional analysis [5], dynamic programming [6,7,8], network flow and mixed-integer linear programming $[9,10,11,12]$, nonlinearprogramming [13], progressive optimality algorithm [14,15], Lagrangian relaxation method [16-18] and modern heuristics algorithms such as artificial neuralnetworks [19], evolutionary algorithm [20-22], chaotic optimization [23], ant colony [24], Tabusearch [25], Expert Systems [26] and simulated annealing [27]. But these methods have one or another drawback such as dimensionality difficulties, large memory requirement or an inability to handle nonlinear characteristics, premature phenomena and trapping into local optimum, taking too much computation time [2].

\section{Optimization Methods and Algorithms}

Hydrothermal scheduling of a power system is concerned with thermal unit commitment and dispatch, and the hourly generation of hydro units [16]. Over two decades, techniques have been developed and results obtained by using the Lagrangian relaxation technique for generating near optimal solution [28,29,30,31]. According to Yan et al[16], Lagrangian relaxation technique decomposes the problem into the scheduling of individual thermal units and the scheduling of individual watersheds, the disadvantage is that the dual solution is generally infeasible [29]. A heuristic method was developed by Yan et al [16] to generate a good feasible solution based on dual results. After the feasible solution is obtained, a few more high level iterations are carried out to obtain additional feasible solutions and the best feasible solution is selected. The final feasible cost and the maximum dual function value are used to calculate the dual gap, a measure of the quality of the feasible schedule.This method did not incorporate pumped-storage unit and cpu time was about four to five minutes.

Extended differential dynamic programming (EDDP) and mixed coordination technique was employed by [8]. The problem was decomposed into a thermal subproblem and hydro subproblem. The thermal subproblem was solved analytically and the hydro subproblem was further decomposed into a set of smaller problems that can be solved in parallel.It was also used to handle unpredictable changes in natural flow.

Zhang et al[32] presented a bundle trust region method (BTRM) to update multipliers within the Lagrangian relaxation framework. Lagrangian multipliers are usually updated by the subgradient method $[33,32]$ which suffers from slow convergence caused by the non-differentiable characteristics of dual functions [32]. The bundle-type methods have been used to update multipliers and are reported in [34,35]. Problem formulation in this case was not split into dual sub-problems but three, I thermal units, J hydro units and Kpumped-storage units with an objective to minimize the total generation cost subject to system-wide demand and reserved requirements, and individual unit constraints. A hierarchical structure of the algorithm is presented 


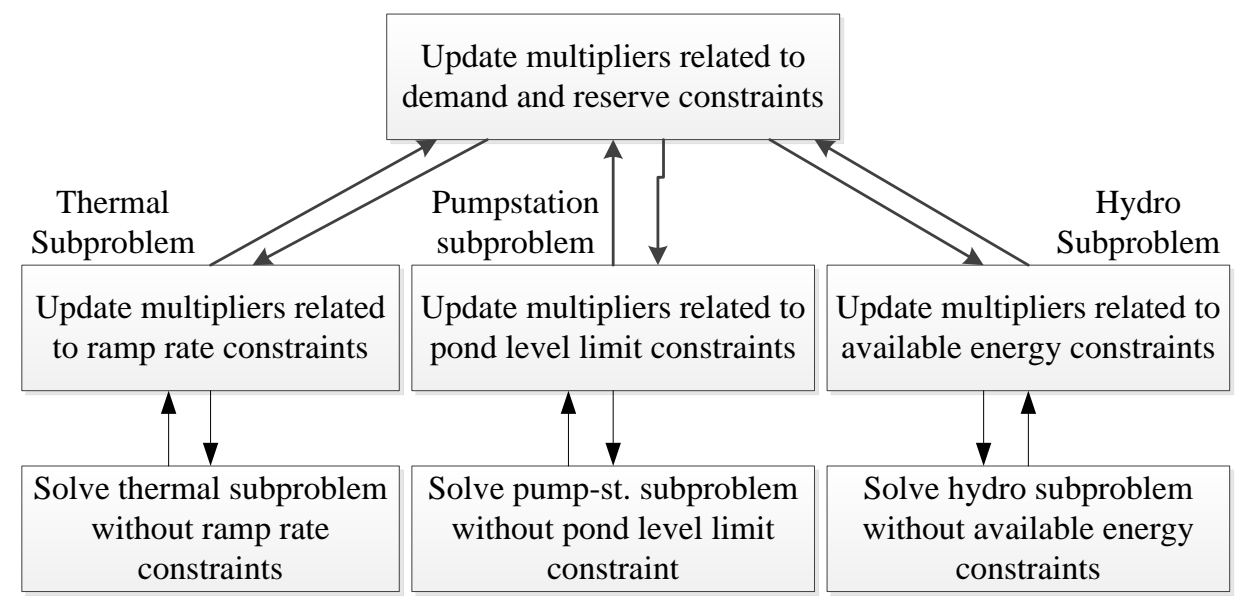

Figure 1 The Hierarchical Structure of the Algorithm [32]

Mendes et al [36] inquired into an algorithm for dual variable updating, usingconcepts of trust region and subgradient algorithms in order to improve both dual optimality and planfeasibility. Comparing with a conventional subgradient algorithm the limitation of using the developed algorithm was: the need to have a small oscillation of the dual function in a neighborhood of the optimal point for the dual problem. The advantages of using the developed algorithm are: good adaptation of step sizes as opposed to trial and error tuning of the parameters in conventional subgradient algorithms.

More recently, a report in 2002 by Nurnberg and Romisch [37] presented a two-stage stochastic programming model for the short or mid-term cost-optimal electric power production planning considering the power generation in a hydro-thermal generation system under uncertainty in demand (or load) and prices for fuel and delivery contracts.The algorithmic approach consisted of a stochastic version of the classical Lagrangian relaxation idea [38], which is very popular in power optimization [39-44].The corresponding coupling constraints contained random variables, hence stochastic multipliers were needed for the dualization, and the dual problem represents a nondifferentiable stochastic program. Subsequently, the approach was based on the same, but stochastic, ingredients as in the classical case: a solver for the nondifferentiable dual, subproblem solvers, and a Lagrangian heuristics. It turns out that, due to the availability of a state-of-the- art bundle method for solving the dual, efficient stochastic subproblem solvers based on a specific descent algorithm and stochastic dynamic programming, respectively, and a specific Lagrangian heuristics for determining a nearly optimal primal solution, this stochastic Lagrangian relaxation algorithm becomes efficient [37].

Gil et al [45] proposed a new model to deal with the short-term generation scheduling problem for hydrothermal systems using genetic algorithms (GAs), a metaheuristic technique inspired on genetics and evolution theories [46]. During the last decade, it has been successfully applied to diverse power systems problems: optimal design of control systems [47,48]; load forecasting [49]; OPF in systems with FACTS [5052]; FACTS allocation [53]; networks expansion [54-56]; reactive power planning [57-59]; maintenance scheduling [60,61]; economic loaddispatch [62,63]; generation scheduling and its subproblems [64-69]. The model handles simultaneously the subproblems of short-term hydrothermal coordination, unit commitment, and economic load dispatch. According to Gil [45] HGSP involves three main decision stages usually separated using a time hierarchical decomposition (Fig. 2): the hydrothermal coordination problem (HCP), the unit commitment problem (UCP), and the economic load dispatch problem (ELDP).

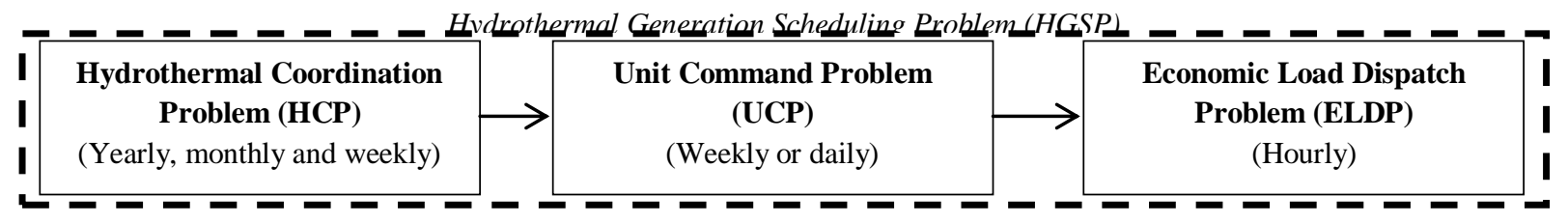

Figure 2 Time hierarchical decomposition for the HGSP [45].

A scheme of the model as presented in Fig. 3, uses as input information, the FCF obtained from a long/mid-term model, detailed information on the hourly load demand, the reservoir inflows and water losses, models of the hydro and thermal generating units and initial conditions, among others. The model uses this input information, handling simultaneously the subproblems of short-term hydrothermal coordination, unit commitment, and economic load dispatch. Considering an analysis horizon period of a week, the model obtains hourly generation schedules for each of the hydro and thermal units [45]. 


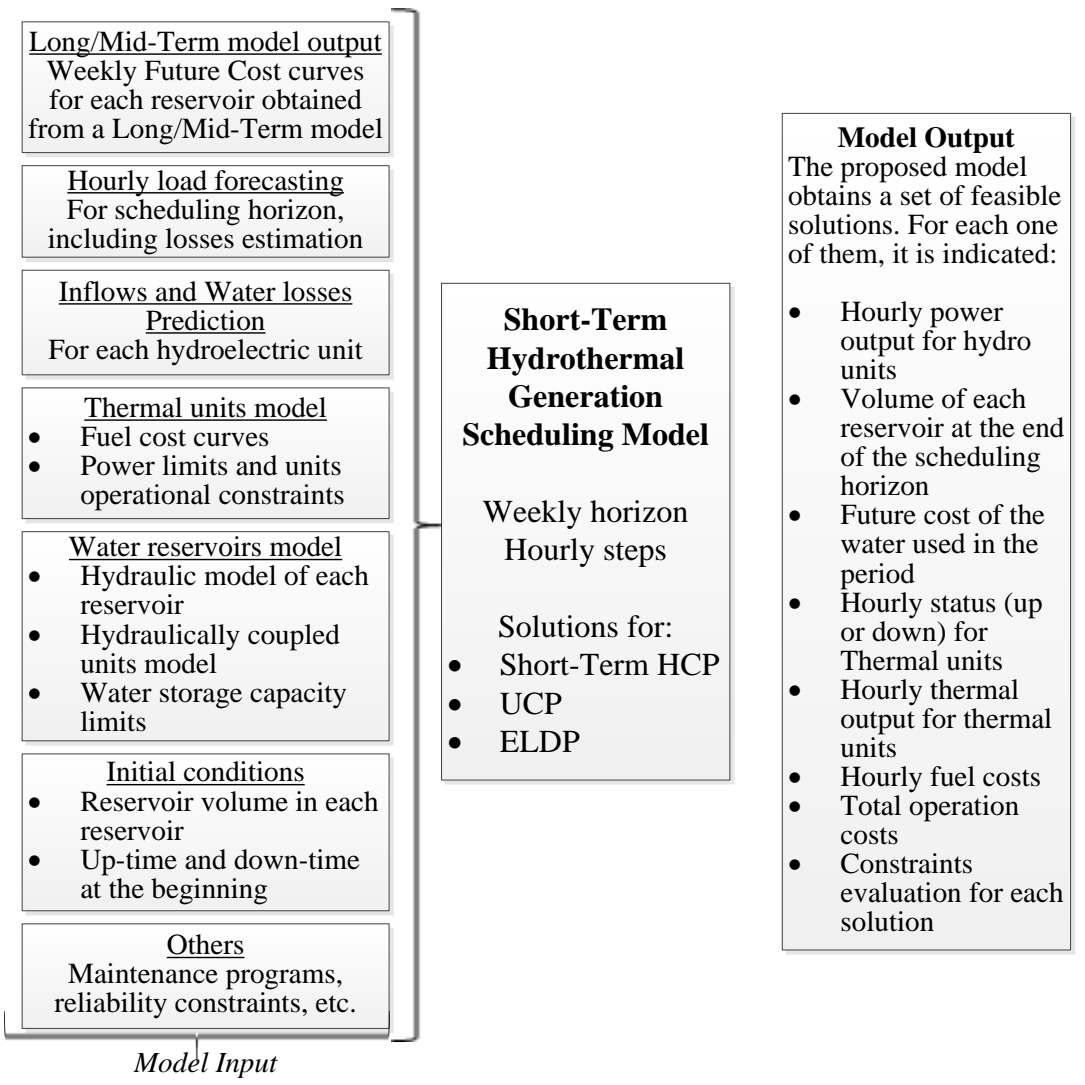

Figure 3 Scheduling model using a Genetic algorithm [45]

Yuan et al [2] proposed a new enhanced cultural algorithm (ECA) to solve the short-term generation scheduling of hydrothermal systems. Cultural algorithm proposed by Reynolds in 1994 [70] is a technique that incorporates domain knowledge obtained during the evolutionary process so as to make the search process more efficient. $\mathrm{CA}$ is a technique that adds domain knowledge to evolutionary computation methods. It is based on the assumption that domain knowledge can be extracted during the evolutionary process by means of the evaluation of each point generated. This process of extraction and use of information has been shown to be effective in decreasing computational cost while approximating global optima in optimization problems[2]. It has been successfully applied to solve optimization problems [71]

The procedure of the proposed ECA[2] for solving the short term generation scheduling of hydrothermal systems is described as follows.

Step 1: $\quad$ Set ECA algorithm parameters and input hydrothermal systems data.

Step 2: Initialize individual solution of water discharge vector $\mathrm{Q}$ for each hydro plant over the scheduling period in population space. Each individual randomly generated is coded using real numbers within their bounds constraint.

Step 3: Calculate storage of the reservoirs over the scheduling period using current values of water discharge.

Step 4: Using water discharge and storage, determine power output of each hydro plant over the scheduling period.

Step 5: Calculate thermal power generation using load balance over the scheduling period, and then evaluate the objective function value (total cost of thermal generation).

Step 6: Evaluate constraint violations using current values of discharge, storage and thermal powers over the scheduling period.

Step 7: Initialize the situational knowledge in belief space. According to the initial water discharge individuals $\mathrm{Q}$ in population space, find the best individual and set as initial situational knowledge.

Step 8: Initialize the normative knowledge in belief space. Let $l_{i}$ and $u_{i}$ be set as the lower and upper bounds for the $i$ th water discharge decision variable, respectively.

Step 9: For each individual in the population space, apply the mutation operator of differential evolution influenced by a randomly chosen situational knowledge and normative knowledge and generate new offspring individual.

Step 10: According to Steps 3-6, evaluate the offspring individual generated.

Step 11: Based on the constraint handling mechanisms, replace the individual with the offspring, if the offspring is better 
Step 12: Update situational knowledge in belief space with the accepted individuals.

Step 13: Update normative knowledge in belief space with the accepted individuals.

Step 14: Check the termination condition. If the maximum iteration number is reached, then obtain the optimal results and stop. Otherwise, go to step 9.

To validate the results obtained with the proposed ECA method, the same problem was solved using a genetic algorithm (GA) and differential evolution (DE). The problems were also solved using the augmented Lagrange method (ALM) and two phase neural network algorithm (TNN). Comparison of results showed that the porposed ECA method can find a lower thermal plant total cost and a faster computation time then the other methods, yields better results while satisfying various constraints. Convergence property of the ECA method is better than that of DE and GA for the solving short-term generation scheduling of hydrothermal systems. The main reason is that the ECA method has a belief space and it can utilize sufficiently the problem-based domain knowledge obtained during the evolutionary process to make the search process more efficient, while DE and GA are lacking this mechanism and thus make its search performance inferior to ECA [2].

Particle swarm optimization (PSO) is a computation technique [72] and has been successfully used in many areas [73-76]. Although PSO has many advantages it has some shortcomings such as premature convergence [77]. To overcome these problems, many methods have been developed, among them is the inertia weight method [78,79]. This could improve the algorithm but does not truly reflect the actual search process to find the optimum. Chang[77] proposed a fuzzy adaptive particle swarm optimization (FAPSO) to the operation of hydro-thermal power system designed to adjust the inertia weight as the environment changes. Fig 4 presents the flowchart of operations for the FAPSO. Chang [77] reports that FAPSO generates better solutions than other methods, mainly because it is implemented to dynamically adjust the inertia weight by using "IF-THEN" rules, this can improve the global and local search ability of the PSO and overcome the disadvantages of the PSO.

Although almost all works which use dual decomposition in the Short-Term Scheduling (STS) problem are based on either Lagrangian Relaxation (LR) [42, 80-85] or Augmented Lagrangian (AL) [86-88], Rodrigues et al[89] proposed a two-phase approach similar to [90,91], but introduced contributions. In the first phase, LR was used to obtain (Infeasible) primal solution, and in the second phase the AL was used to obtain a solution whose quality can be assessed by the LR. Aiming to deal with nonlinearities and binary variables of hydrosystems, the LR was used but including the AL second-phase optimization in order to achieve primal feasibility, modeling all nonlinearities and binary variables related to hydro- and thermal units. The infeasibility between generation and demand is eliminated and the artificial constraints are satisfied.

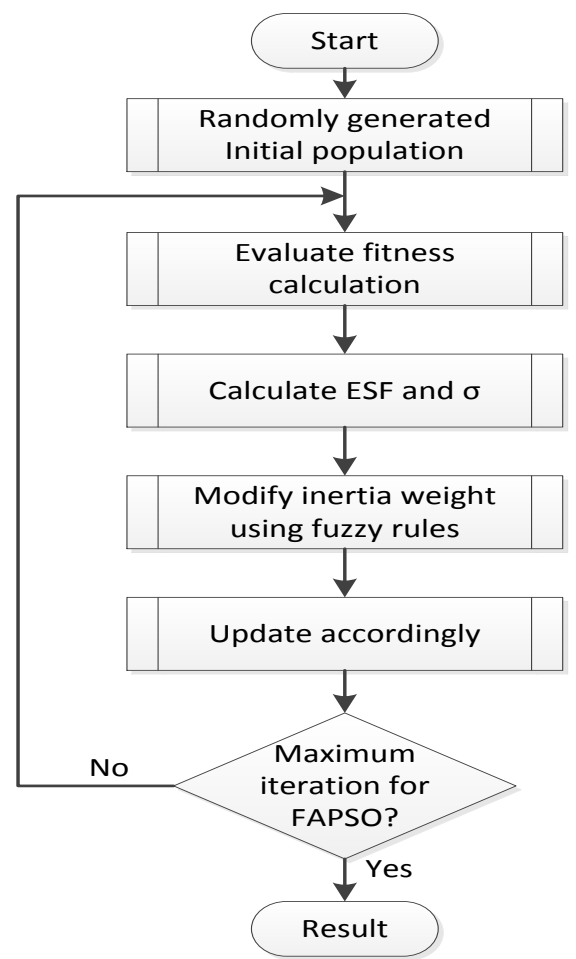

Figure 4 The framework flow chart of FAPSO [77].

Typically, LR technique relaxes coupling constraints such as demand and reserve requirements. Nevertheless, using this strategy, the hydro-subproblem remains coupled in time and space [89]. An alternative approach consists in combining LR with Variable Splitting-LRVS method [92,44], where the decomposition is achieved 
by duplicating some variables. Rodrigues et al [89]used the LRVS to duplicate thermal and hydrovariables, as well as the turbined outflow and spillage variables, thusobtaining four subproblems: thermal, hydro, hydrothermal, and hydraulic. The first two subproblems take into account the unit commitment constraints (thermal and hydro). The hydrothermal subproblem considers demand reserve and transmission constraints. Finally, all the reservoir constraints are modelled into the hydraulic subproblem.Given that the LRVS fails to find a feasible solution, an AL approach was used in attempt to overcome this issue. The artificial constraints relaxed in the LRVS are taken into account in the AL function. In order to maintain the decomposition, the Auxiliary Problem Principle (APP) [93] was employed. In terms of solution strategy, the pseudoprimal point strategy [94]was included as a warm starting by the APP. Thisstrategy improves dramatically the performance of the APP algorithm.

\section{Discussion}

According to Atul [1], the operating cost of thermal plant is very high, though their capital cost is low. On the other hand, the operating cost of hydroelectric plant is low, though their capital cost is high, so it has become economical as well as convenient to have both thermal and hydro plants in the same grid.The objective of optimal operation to hydrothermal power is usually to minimize the thermal cost function while satisfying physical and operational constraints [77].

Intensive reviewing shows that hydrothermal generation scheduling problem has to be decomposed into smaller problems in order to solve it [95]. Since the Lagrangian relaxation (LR) approach was proposed in [96] for solving the problem of optimal short-term resource scheduling in large-scale power systems it has been recognized as a good approach $[97,85]$. One of the most appealing properties of the LR approach is its linearity between the computing time and the number of resources, its convenience to handle resource-specific constraints and its estimation of the quality of the solution, that is, the duality gap. The LR approach can be interpreted as a two-stage hierarchical process, a decomposition stage and a coordination stage. The last stage consists in solving an optimization problem called dual problem, involving the updating of the dual variables [36]. One optimization techniques to solve the dual problem has been the usual subgradient algorithm. Itsuffers from some drawbacks, the selection of the rulefor the step size is one crucial problem [36] and suffers from slow convergence caused by the non-differentiable characteristics of dual functions [32]. The main advantages of the LR are as follows: the original problem can be split into a sequence of smaller easy-to-solve subproblems, and a lower-bound for the optimal objective function is supplied. However, there is an important disadvantage: for nonconvex problems, the LR fails to find a feasible solution. On the other hand, with AL, it is possible to obtain a feasible solution or a near-feasible solution [89].

Convergence property of the ECA method is better than that of DE and GA for solving short-term generation scheduling of hydrothermal systems. The main reason is that the ECA method has a belief space and it can utilize sufficiently the problem-based domain knowledge obtained during the evolutionary process to make the search process more efficient, while DE and GA are lacking this mechanism and thus make its search performance inferior to ECA [2].

Based on this review, the fuzzy adaptive particle swarm optimization (FAPSO) [77] and the Lagrangian and Augmented Lagrangian [89] methods produced faster convergence and efficient optimization schemes.

\section{Conclusion}

All the techniques reviewed and methods presented claim to have faster convergence time and reduced CPU speed. After intensive reviewing, it is unsurprising that, certainly new efficient methods are developed continually to increase computation speed and achieve near ideal convergence for optimal schedule of hydrothermal systems. In this review, the two most recent articles[77] and [89] are efficient models that attempted to consider all factors in the problems of scheduling hydrothermal systems. It is hoped that this paper exposes to its audience the studies carried out in optimization of hydrothermal systems.

\section{References}

[1] K. S. Atul, "Short Term Hydrothermal Scheduling using Evolutionary Programming," Electrical \& Instrumentation Engineering Department, Thapar University, Patiala, Master of Engineering Thesis in Power Systems \& Electric Drives (Supervised by Mr. Nitin Narang) 2009.

[2] X. Yuan, H. Nie, Y. Yuan, A. Su, and L. Wang, "Hydrothermal systems generation scheduling using cultural algorithm.," ournal of Hydroinformatics, vol. 11, no. 1, pp. 65-78, 2009.

[3] J. Grake and L. Kirchmayer, "Optimum operation of a hydrothermal system.," AIEE Trans., vol. PAS 80, pp. $242-250,1962$.

[4] M. Papageorgiou, "Optimum mult reservoir network control by the discrete maximum principle.," Water Resour. Res., vol. 21, no. 2, pp. 1824-1830, 1985.

[5] S. Solima and G. Christensen, "Application of functional analysis to optimization of variable head multi reservoir power system for long term regulation.," Water Resour. Res., vol. 22, no. 6, pp. 852-858, 1986. 
[6] J. Yang and N. Chen, "Short-term hydrothermal coordination using multipass dynamic programming.," IEEE Transactions on Power Systems, vol. 4, no. 3, pp. 1050-1056, 1989.

[7] W. Yeh, "Optimization of real time hydrothermal system operation," J. Water Res. Plan. Manage., vol. 118, no. 6, pp. 636-653, 1992.

[8] J. Tang and P.B. Luh, "Hydrothermal Scheduling Via Extended Differential Dynamic Programmng and Mixed Coordination*," IEEE Transaction on Power Systems, vol. 10, no. 4, pp. 2021-2028, November 1995.

[9] P. Franco, M. Carvalho, and S. Soares, "A network flow model for short-term hydro-dominated hydrothermal scheduling problems.," IEEE Trans. Power Syst., vol. 9, no. 2, pp. 1016-1022, 1994.

[10] M. Piekutowski, "Optimal short-term scheduling for large-scal cascaded hydro system.," IEEE Trans. Powr Syst., vol. 9, no. 2, pp. $805-811,1994$.

[11] G. Oliveira and S. Soares, "A second order network flow algorithm for hydrothermal scheduling.," IEEE Trans. Power Syst., vol. 10, no. 3, pp. 1635-1641, 1995.

[12] G. Chang, M. Aganagic, and J. Waight, "Experiences with mixed integer linear programming based approaches on short-term hydro scheduling.," IEEE Trans. Power Syst, vol. 16, no. 4, pp. 743-749, 2001.

[13] X. Guan and P.B. Luh, "Nonlinear approximation method in Lagrangian relaxation-based algorithms for hydrothermal scheduling.," IEEE Trans. Power Syst., vol. 10, no. 2, pp. 772-778, 1995.

[14] A. Turgeon, "Optimal short-term hydro scheduling from the principle of progressive optimality.," Water Resour. Res., vol. 17, no. 3, pp. 481-486, 1981.

[15] F. Lee, "A method to eliminate solution trapping in applying progressive optimality principle to short-term hydrothermal scheduling.," IEEE Trans. Power Syst., vol. 4, no. 3, pp. 935-942, 1989.

[16] H. Yan, P.B. Luh, X. Guan, and P.M. Rogan, "Scheduling of Hydrothermal Power Systems," IEEE Transactions on Power Systems, vol. 8, no. 3, pp. 1358-1365, August 1993.

[17] N. Tufegdzic, "A coordinated approach for real-time short-term hydro scheduling.," IEEE Trans. Power Syst., vol. 11, no. 4, pp. 1698-1704, 1996.

[18] M. Salam and K. Modamed, "Hydrothermal scheduling based Langrangian relaxation approach to hydrothermal coordination.," IEEE Trans. Power Syst., vol. 13, no. 1, pp. 226-235, 1998.

[19] R. Naresh and J. Sharma, "Two-phase neural network based solution technique for short term hydrothermal scheduling.," IEE Proc. Gener. Transm. Distrib., vol. 146, no. 6, pp. 657-663, 1999.

[20] P. Yang and H. Yang, "Scheduling short-term hydrothermal geenration using evolutionary programming techniques.," IEE Proc. Gener. Transm. Distrib., vol. 143, no. 4, pp. 65-72, 1996.

[21] S. Orero and M. Irving, "A genetic algorithm modeling framework and solution technique for short term optimal hydrothermal scheduling.," IEEE Trans. Power Syst., vol. 13, no. 2, pp. 501-518, 1998.

[22] T. Werner and J. Verstege, "An evolution strategy for short-term operation planning of hydrothermal systems.," IEEE Trans. Power Syst., vol. 14, no. 4, pp. 1362-1368, 1999.

[23] X. Yuan and Y. Yuan, "A hybrid chaotic genetic algorithm for short-term hydro system scheduling.," Math. Comput. Simul., vol. 59, no. 4, pp. 319-327, 2002.

[24] S. Huang, "Enhancement of hydroelectric generation scheduling using ant colony system based optimization approaches.," IEEE Trans. Energy Convers., vol. 16, no. 3, pp. 296-301, 2001.

[25] X. Bai and S. Shahidehpour, "Hydro-thermal scheduling by tabu search and decompoisition method.," IEEE Trans. Power Syst., vol. 11, no. 2, pp. 968-974, 1996.

[26] S. Li, S.M. Shahidehpour, and C. Wang, "Promoting the application of Expert Systems in Short-Term Commitment.," IEEE Transactions on Power System., vol. 3, no. 1, pp. 286-292, May 1993.

[27] K. Wong and Y. Wong, "Short-term hydrothermal scheduling Part 1: simulated annealing approach.," IEE Proc. Gener. Transm. Distrib., vol. 141, no. 5, pp. 497-501, 1994.

[28] A. Cohen, "Optimization-Based Methods for Operations Scheduling," Proceedings of IEE, vol. 75, no. 12, pp. $1574-1591,1987$.

[29] L.A.F.M. Ferreira et al., "Short-Term Resource Scheduling in Multi-Area Hydrothermal Power Systems," Electric Power \& Energy Systems, vol. 11, no. 3, pp. 200-212, July 1989.

[30] M.V. Rakic, M.L. Markovic, and Z.M. Markovic, "Short-Term Operation Planning of Combined Power Systems: A Decomposition Technique.," in Proceedings of Tenth Power Systems Computation Conference, Graz, Austria, 1990, pp. 1155-1162.

[31] S. Soares, C. Lyra, and H. Tavares, "Optimal Generation Scheduling of Hydrothermal Power Systems.," IEEE Transactions on Power Apparatus and Systems, vol. PAS -99, no. 3, pp. 1107-1115, May/June 1980.

[32] D. Zhang, P.B. Luh, and Y. Zhang, "A Bundle Method for Hydrothermal Scheduling," IEEE Transactions on Power Systems, vol. 14, no. 4, pp. 1355 - 1361, November 1999.

[33] G.L. Nemhauser and L.A. Wolsey, Integer and Combinatorial Optimization.: John Wiley \& Sons, 1988.

[34] F. Pellegrino, A. Renaud, and T. Socroun, "Bundle and Augmented Lagrangian Method for Short Term Unit Commitment," in Proceedings of 12th Power System Computation Conference, Dresden, 1996.

[35] V. Mendes, L. Ferreira, P. Roldao, and R. Pestana, "Optimal Short Term Resource Scheduling by Lagrangian Relaxation: Bundle Type Versus Subgradient Algorithms.," in Proceedings of 12th Power System Computation Conference, Dresden, 1996.

[36] V.M.F. Mendes, L.A.F.M. Ferreira, P. Roldao, and R. Pestana, "Short-term Resource Scheduling in Hydrothermal Power System by Lagrangian Relaxation: An Approach for Dual Variable Updating".

[37] R. Nurnberg and W. Romisch, "A Two-Stage Planning Model for Power Scheduling," Optimization and Engineering, vol. 3, pp. 355$378,2002$.

[38] C. Lemarechal, "Lagrangian decomposition and nonsmooth optimization," in Nonsmooth Optimization Methods and Applications, F. Gianessi, Ed.: Gordon and Breach, 1992, pp. 201-216.

[39] D. P. Bertsekas, G. S. Lauer, N. R. Sandell Jr., and T. A. Posbergh, "Optimal short-term scheduling of large-scale power systems," IEEE Transactions on Automatic Control, vol. AC-28, pp. 1-11, 1983.

[40] F. Zhuang and F. D. Galiana, "Towards a more rigorous and practical unit commitment by Lagrangian relaxation," IEEE Transactions on Power Systems, vol. 3, pp. 763-773, 1988.

[41] G. B. Sheble and G. N. Fahd, "Unit commitment literature synopsis," IEEE Transactions on Power Systems, vol. 9, pp. 128-135, 1994.

[42] R. Gollmer, A. Moller, M. P. Nowak, W. Romisch, and R. Schultz, "Primal and dual methods for unit commitment in a hydrothermal power system," in Proceedings of 13th Power Systems Computation Conference, Trondheim, Norway, 1999, pp. 724-730.

[43] S. Feltenmark and K.C. Kiwiel, "Dual applications of proximal bundle methods, including Lagrangian relaxation of nonconvex problem," SIAM Journal on Optimization, vol. 10, pp. 697-721, 2000. 
[44] C. Lemarechal and A. Renaud, "A geometric study of duality gaps, with applications," Mathematical Programming, vol. 90, no. 3, pp. 399-427, 2001.

[45] E. Gil, J. Bustos, and H. Rudnick, "Short-term Hydrothermal Generation Scheduling Model Using a Genetic Algorithm," IEEE Transaction on Power Systems, vol. 18, no. 4, pp. 1256-1264, 2003.

[46] D.E. Goldberg, Genetci Algorithms in Search, Optimization, and Machine Learning. Reading, MA: Addison-Wesley, 1989.

[47] R. Dimeo and K.Y. Lee, "Boiler-turbine control system design using a genetic algorithm.," IEEE Trans. Energy Conversion, vol. 10, pp. 752-759, December 1995.

[48] R.A.F. Saleh and H.R. Bolton, "Genetic algorithm-aided design of a fuzzy logic stabilizer for a superconducting generator.," IEEE Transactions on Power System, vol. 15, pp. 1329-1335, November 2000.

[49] T. Maifeld and G. Sheble, "Short-term load forecasting b a neural network and a refined genetic algorithm," Electric Power Syst. Res., vol. 31, no. 3, pp. 147-152, December 1994.

[50] N. Li, Y. Xu, and H. Chen, "FACTS-based power flow control in interconnected power system.," IEEE Transactions on Power Sysems, vol. 15, pp. 257-262, February 2000.

[51] T.S. Chung and Y.Z. Li, "A hybrid approach for OPF with consideration of FACTS devices.," IEEE Power Eng. Rev., vol. 20, pp. 54-57, August 2000.

[52] L.L. Lai and J.T. Ma, "Genetic algorithms and UPFC for power flow control," Int. J. Eng. Intell. Syst., vol. 4, pp. 237-242, December 1996.

[53] S. Gerbex, R. Cherkaoui, and A.J. Germond, "Optimal location of multi-type FACTS devices in a power system by means of genetic algorithms.," IEEE Transactions on Power Systems, vol. 16, pp. 537-544, August 2001.

[54] V. Miranda, J.V. Ranito, and L.M. Proença, "Genetic algorithm in optimal multistage distribution network planning," IEEE Trans. Power Syst., vol. 9, pp. 1927-1933, November 1994.

[55] H. Rudnick, R. Palma, E. Cura, and Silva, "Economically adapted transmission systems in open access schemes: Application of genetic algorithm," IEEE Trans. Power Syst., vol. 11, pp. 1427-1440, August 1996.

[56] R.A. Gallego, A.J. Monticelli, and R. Romero, "Comparative studies on nonconvex optimization methods for transmission network expansion planning," IEEE Trans. Power Syst., vol. 13, pp. 822-828, August 1998.

[57] K. Iba, "Reactive power optimization by genetic algorithm," IEEE Trans. Power Syst., vol. 9, pp. 685-692, May 1994.

[58] K.Y. Lee, X. Bai, and Y.M. Park, "Optimization method for reactive power planning by using a modified simple genetic algorithm," IEEE Transactions on Power Systems, vol. 10, pp. 1843-1850, November 1995.

[59] K.Y., Yang, F.F. Lee, "Optimal reactive power planning using evolutionary algorithms: A comparative study for evolutionary programming, evolutionary strategy, genetic algorithm, and linear programming," IEEE Trans. Power Syst., vol. 13, pp. 101-108, February 1998.

[60] A. Ahmad and D.P. Kothari, "A practical model for generator maintenance scheduling with transmission constraints," Electric Mach. Power Syst., vol. 28, no. 6, p. 501=513, June 2000

[61] I. El-Amin, S. Duffuaa, and M. Abbas, "A Tabu search algorithm for maintenance scheduling of generating units," Electric Power Syst. Res., vol. 54, no. 2, pp. 91-99, May 2000.

[62] H.K. Youssef and K.M. El-Naggar, "Genetic based algorithm for security constrained power system economic dispatch.," Electric Power Syst, Res., vol. 53, no. 1, pp. 47-51, January 2000.

[63] T. Jayabarathi, G. Sadasivam, and V. Ramachandram, "Evolutionary programming-based multiarea economic dispatch with tie line constraints.," Electric Mach. Power Syst., vol. 28, no. 12, pp. 1165-1176, December 2000.

[64] Y.-G. Wu, C.-Y. Ho, and Wang D.-Y., "A diploid genetic approach to short-term scheduling of hydrothermal system," IEEE Trans. Power Syst., vol. 15, pp. 1268-1274, November 2000.

[65] C.-P. Cheng, C.-W. Liu, and C. Liu, "Unit commitment by Lagrangian relaxation and genetic algorithms," IEEE Trans. Power Syst., vol. 15, pp. 707-714, May 2000

[66] C. W. Richter Jr. and G. B. Sheblé, "A profit-based unit commitment GA for the competitive environment," EEE Trans. Power Syst., vol. 15, pp. 715-721, May 2000.

[67] K. P. Wong and Y. W. Wong, "Combined genetic algorithm/simulated annealing/fuzzy set approach to short-term generation scheduling with take-or-pay contract.," IEEE Trans. Power Syst., vol. 11, pp. 128-136, February 1996.

[68] A. A. El Desouky and M. M. Elkateb, "A hybrid artificial intelligence and heuristic method to short term generation scheduling," in Proc. Int., Marbella, Spain, 2000, pp. 147-152

[69] J. Valenzuela and A.E. Smith, "A seeded memetic algorithm for large unit commitment problems.," J. Heuristics, vol. 8, no. 2, pp. 173-195, 2002.

[70] R. Reynolds, "An introduction to cultural algorithms.," in Proceedings of 3rd Annual Conference on Evolutionary Programming., Singapore, 1994, pp. 131-139.

[71] X. Yuan and Y. Yuan, "Application of cultural algorithm to generation scheduling of hydrothermal systems.," Energy Convers. Manage., vol. 47, no. 15, pp. 2192-2201, 2006.

[72] J. Kennedy and R. Eberhart, "Particle Swarm Optimization.," in Proceedings of IEEE International COnference Neural Networks., vol. 4, Perth, 1995, pp. 1942-1948.

[73] Z.L. Gaing, "A Particle Swarm Optimization Approach for Optimum Design of PID Controller in AVR System.," IEEE Transactions on Energy Conversion., vol. 19, no. 2, pp. 384-391, June 2004.

[74] B. Zhao, C.X. Guo, and Y.J. Cao, "A Multiagent-Based Particle Swarm Optimization Approach for Optimal Re-active Power Dispatch.," IEEE Transactions on PowerSystem, vol. 20, no. 2, pp. 1070-1078, May 2005.

[75] I. Selvakunar and K. Thanushkodi, "A New Particle Swarm Optimization Solution to Nonconvex Economic Dispatch Problems.," IEEE Transactions on Power System., vol. 22, no. 1, pp. 42-51, February 2007.

[76] K.T. Charturvedi, M. Pandit, and L. Srivastava, "Self-Organizing Hierachical Particle Swarm Optimization for Nonconvex Economic Dispatch.," IEEE Transactions on Power System., vol. 23, no. 3, pp. 1079-1087, August 2008.

[77] W. Chang, "A Novel Particle Swarm Optimization for Optimal Scheduling of Hydrothermal System.," Energy and Power Engineering., vol. 2, pp. 223-229, November 2010.

[78] Y. Shi and R. Eberhart, "A Modified Particle Swarm Optimize," Evolutionary Computation Proceedings, IEEE Congress on Computational Intelligence., pp. 69-73, 1998.

[79] Y. Shi and R. Eberhart, "Empirical Study of Particle Swarm Optimization.," in IEE Congress on Evolutionary Computation, 1999, pp. 1949-1950.

[80] E. C. Finardi and E. L. da Silva, "Solving the hydro unit commitment problem via dual decomposition and sequential quadratic programming," IEEE Transactions on Power Systems, vol. 21, no. 2, pp. 835-844, 2006. 
[81] C.A. Li, E. Hsu, A. J. Svoboda, C. L. Tseng, and R. B. Johnson, "Hydro unit commitment in hydrothermal optimization," IEEE Transactions on Power Systems, vol. 12, no. 2, pp. 764-769, 1997.

[82] A. Borghetti, A. Frangioni, F. Lacalandra, and C.A. Nucci, "Lagrangian heuristics based on disaggregated Bundle methods for hydrothermal unit commitment," IEEE Transactions on Power Systems, vol. 18, no. 1, pp. 313-323, 2003.

[83] S. Takriti and J.R. Birge, "Using integer programming to refine Lagrangian-based unit commitment solutions.," IEEE Transactions on Power Systems., vol. 15, no. 1, pp. 151-156, 2000.

[84] S.Y. Lai and R. Baldick, "Unit commitment with ramp multipliers.," IEEE Transactions on Power Systems., vol. 14, no. 1, pp. 58-64, 1999.

[85] N.J. Redondo and A.J. Conejo, "Short-term hydro-thermal coordination by lagrangian relaxation: solution of the dual problem," IEEE Transactions on Power Systems., vol. 14, no. 1, pp. 89-95, Month 1999.

[86] S.J. Wang, S.M. Shahidehpour, D.S. Kirschen, S. Mokhtari, and G.D. Irisarri, "Short-term generation scheduling with transmission and environmental constraints using an augmented Lagrangian relaxation," IEEE Transactions on Power Systems, vol. 10, no. 3, pp. 1294-1301, 1995.

[87] S. Al-Agtash and R. Su, "Augmented lagrangian approach to hydro-thermal scheduling," IEEE Transactions on Power Systems., vol. 13, no. 4, pp. 1392-1400, 1998.

[88] S. Al-Agtash, "Hydrothermal scheduling by augmented Lagrangian: consideration of transmission constraints and pumped-storage units," IEEE Transactions on Power Systems, vol. 16, no. 4, pp. 750-756, 2001

[89] R.N. Rodrigues, E.L. da Silva, E.C. Finardi, and Y.K. Takigawa, "Solving the Short-Term Scheduling Problem of Hydrothermal Systems via Lagrangian Relaxation and Augmented Lagrangian," Mathematical Problems in Engineering, vol. doi:10.1155/2012/856178, p. 18, 2012.

[90] A. Belloni, A. L. D. S. Lima, M. E. P. Maceira, and C. A. Sagastiz'abal, "Bundle relaxation and primal recovery in unit commitment problems-the Brazilian case," Annals of Operations Research, vol. 120, no. 1, pp. 21-44, 2001.

[91] R. N. Rodrigues, E. C. Finardi, and E. L. da Silva, "Solving the short-term scheduling problem of hydrothermal systems via Lagrangian relaxation and augmented Lagrangian," in Proceedings of 16th Power Systems Computation Conference, Glasgow, Scotland, 2008.

[92] O. Nilsson and D. Sjelvgren, "Variable splitting applied to modelling of start-up costs in short term hydro generation scheduling.," IEEE Transactions on Power Systems., vol. 12, no. 2, pp. 770-775, 1997.

[93] C. Cohen, "Auxiliary problem principle and decomposition of optimization problems.," Journal of Optimization Theory and Applications, vol. 32, no. 3, pp. 277-305, 1980.

[94] L. Dubost, R. Gonzalez, and C. Lemarechal, "A Primal-Proximal Heuristic Applied to the Unit-Commitment Problem.," Institut National de Recherche in Informatique et en Automatique, 2003.

[95] A.J. Wood and B.F. Wollenberg, Power Generation, Operation and Control. New York: Wiley, 1996.

[96] J.A. Muckstadt and S.A. Koenig, "An application of Lagrangian relaxation to scheduling in power-generating systems," Operations Research, vol. 25, no. 3, May-June 1977.

[97] A. Merlin and P. Sandrin, "A New Method for Unit Commitment at Electricite De France," IEEE Transaction on Power Systems, vol. PAS-102, no. 5, May 1983. 